\title{
The clinical course and long-term outcome of juvenile systemic lupus erythematosus
}

\author{
M Wierzbowska*, P Gietka, L Rutkowska-Sak and E Musiej-Nowakowska
}

Address: Institute of Rheumatology, Warsaw, Poland

* Corresponding author

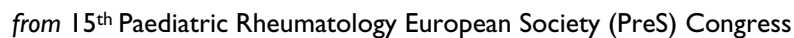

London, UK. 14-17 September 2008

Published: 15 September 2008

Pediatric Rheumatology 2008, 6(Suppl I):P252 doi:I0.II86/I546-0096-6-SI-P252

This abstract is available from: http://www.ped-rheum.com/content/6/SI/P252

(c) 2008 Wierzbowska et al; licensee BioMed Central Ltd.

The aim of the study was to examine the evolution of the clinical features, clinical course of juvenile systemic lupus erythematosus (jSLE) and to estimate most frequent longterm outcome of the disease according to SLICC/ACR Damage Index for SLE in a group of children whose disease began after 1985 and has been hospitalized in Institute of Rheumatology in Warsaw since 1985 till 2005. The study involved an analysis of medical records of 138 patients (119 girls, 19 boys; mean age at the onset of SLE $7,9 \pm 0,2)$. They have been observed for the mean period of $12,5 \pm 0,39$.

The statistical analysis proved that the occurence of clinical and immunological features as well as the primary jSLE activity has been changing during the last twenty years.

The number of clinical jSLE manifestations has also decreased and the mortality rate has fallen. The most frequent cause of death is nowadays generalized infections.

Lower SLE Disease Activity Index (SLEDAI) scores are being observed on the beginning of jSLE.

There is statistically significant less renal involvements and seizures at the onset of jSLE.

Observed growth in frequency of psychosis, ds-DNA antibody presence, hair los and haemolytic aenemia was statistically significant.
Most frequent long-time outcomes of the disease are: nephropaty, central nervous system involvement, vasculopathy and skin changes. 